ENCYCLOPEDDIE Encyclopédie berbère

BERBERE

$23 \mid 2000$

23 | Hiempsal - Icosium

\title{
Huwwâra, Houuara, Houara, Hawwâra
}

(transcriptions qui varient selon l'auteur)

\section{Gast}

\section{OpenEdition}

\section{Journals}

Édition électronique

URL : http://journals.openedition.org/encyclopedieberbere/1612

DOI : 10.4000/encyclopedieberbere.1612

ISSN : 2262-7197

\section{Éditeur}

Peeters Publishers

\section{Édition imprimée}

Date de publication : 1 octobre 2000

Pagination : 3513-3521

ISBN : 2-7449-0207-1

ISSN : $1015-7344$

\section{Référence électronique}

M. Gast, « Huwwâra, Houuara, Houara, Hawwâra », Encyclopédie berbère [En ligne], 23 | 2000

document H61, mis en ligne le 01 juin 2011, consulté le 24 septembre 2020. URL : http://

journals.openedition.org/encyclopedieberbere/1612 ; DOI : https://doi.org/10.4000/ encyclopedieberbere.1612

Ce document a été généré automatiquement le 24 septembre 2020

(c) Tous droits réservés 


\title{
Huwwâra, Houuara, Houara, Hawwâra
}

\author{
(transcriptions qui varient selon l'auteur)
}

\section{Gast}

1 Vocable générique qui désignait un ensemble (peut-être confédéral) de populations situées avant l'arrivée des Arabes, de la Tripolitaine au Fezzan en Libye.

Ibn Khaldoun parle longuement des Huwwâra dans son célèbre ouvrage sur l'Histoire des Berbères. Il dit notamment :

"Lors de la conquête musulmane, toutes les tribus portant le nom générique de Hoouara, tant celles qui remontent leur origine à El-Abter que celles qui ont Bernès pour ancêtre, habitaient la province de Tripoli et la partie du territoire de Barca qui en est voisine; fait que rapportent également El-Masoudi et El-Bekri. Les unes possédaient des demeures fixes, les autres vivaient en nomades. Parmi elles, il s'en trouva une qui traversa les sables jusqu'au Désert et s'établit à côté des Lamta porteurs du voile, qui habitaient auprès de Gaugaua, localité située dans le pays des Noirs, vis à vis de rifrîkïa. On reconnaît l'origine hoouaride de cette peuplade au nom qu'elle porte et qui est une altération du mot Hoouara; car ayant changé le ou de ce mot en une espèce de $k$ dont le son est l'intermédiaire du $\mathrm{k}$ doux et $\mathrm{du} \mathrm{k}$ guttural, ils en ont formé Heggar" (Ibn Khaldoun, Histoire des Berbères, t. 1, 1925 : 275-276).

Nous remarquons que la racine HWR (que de Foucauld note au mot ehouer; Dict. touareg-français p. 1518-19, qui signifie "précéder") apparaît encore aujourd'hui dans l'expression tamâhaq : Ihuwwaraq qui veut dire en Ahaggar : "je suis plus âgé que toi" et par conséquent "tu me dois le respect", d'où la connotation de supériorité. Alors que Ihuwwarahi "il est plus âgé que moi" signale l'obligation de respect, de soumission, de celui qui parle envers le tiers plus âgé. Le terme générique de Huwwâra signifierait donc, par extension, "Suzerains", "Dominants". Il est singulier qu'après plusieurs siècles d'avatars ce vocable, devenu Ahaggar, désigne toujours les suzerains, les "nobles" (de Foucauld nomme ainsi ceux de l'Ahaggar, de l'Ajjer et les Taïtoq). Les généalogistes et historiens arabes se seraient donc trompés en prenant comme nom de personne le vocable désignant d'une façon générale une aristocratie dominante chez ces Berbères. Le nom de Huwwar, fils d'Aurigh, fils de Bernès, ne serait qu'un essai de 
cohérence pour donner une filiation à ceux que l'on avait pris l'habitude d'appeler aussi "enfants de Tiski".

Quand les conquérants arabes, ignorant la langue berbère, demandèrent le nom de ces populations qui s'opposaient à leur passage, on leur répondit probablement "ce sont les Huwwâra", c'est-à-dire "les maîtres du pays", "les suzerains". Car il est malséant de donner à un étranger son nom et celui de sa famille. Ce réflexe de défense a engendré dans toute l'histoire de l'Afrique du Nord de nombreux vocables créés de toutes pièces par les étrangers qui ignoraient en général les langues du pays.

L'on peut imaginer ces fiers Huwwâra habitués à commander les populations de leur environnement, à guerroyer à cheval ou à chameau à l'aide de leurs armes traditionnelles qui devaient être à l'époque l'arc, les javelots de bois à armatures de fer, le bouclier rond, le poignard de bras, vêtus de tuniques de peau, allant tête nue et se nourrissant essentiellement de lait et de viande et qui voient arriver des cavaliers richement harnachés, armés de grands sabres recourbés, vêtus de vêtements de coton et de soie, coiffés de cimiers, de turbans colorés et qui parlent avec une foi inébranlable au nom de l'islam et de son prophète Muhammad! Le choc psychologique, l'humiliation et le dépit qu'ont dû subir ces Dominants qui devaient se soumettre à ces étrangers ou fuir ont été à la mesure de la haine et de la révolte qu'ils ont développées par la suite.

Situation des Howara et pénétration musulmane dans le Bilad al Sudan (d'après Cuoq).

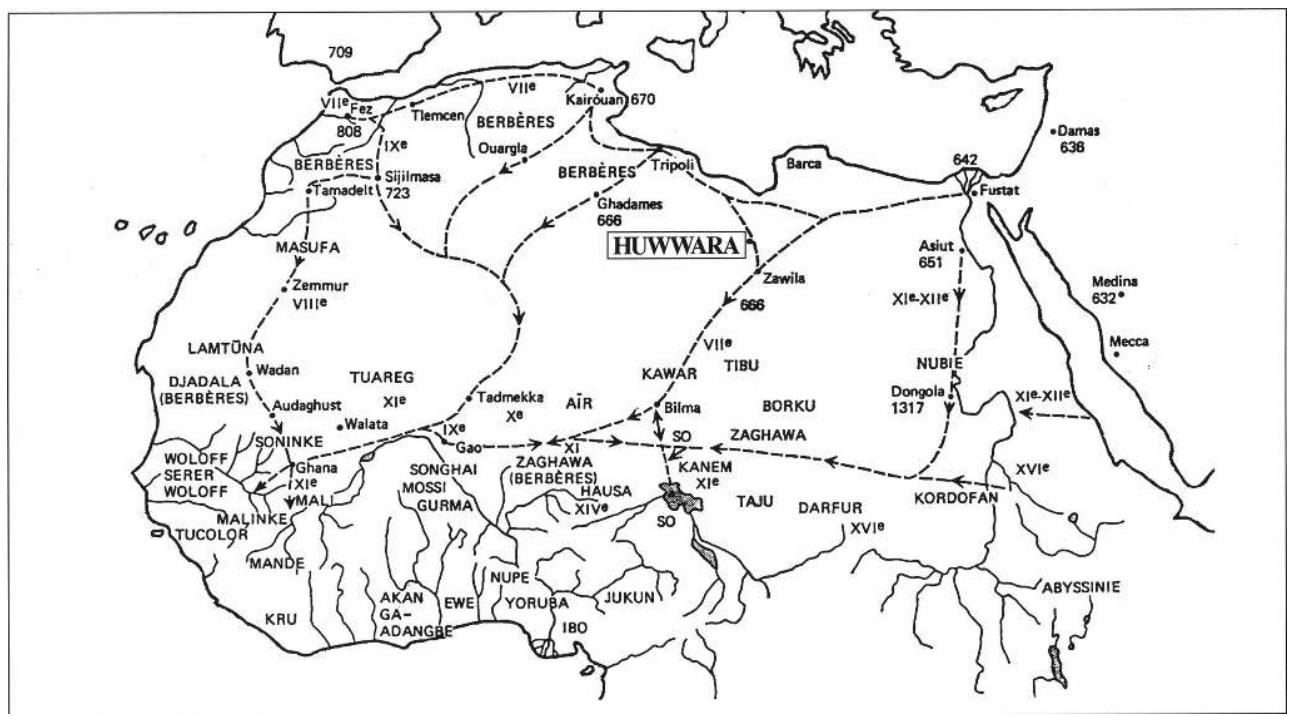

Tout en apprenant d'eux la nouvelle religion (alors qu'ils étaient païens, juifs ou chrétiens), des techniques de combat, une hiérarchie de commandement et d'organisation militaire, ils recherchèrent très vite comment échapper à l'arrogance de leurs conquérants sans subir l'anathème d'impies, car aucun païen déclaré ne pouvait échapper à la mort.

7 La première réaction de tout ce peuple traversé par la fulgurance des troupes arabes a été la fuite à l'ouest, vers des régions dont ils connaissaient bien les itinéraires, mais aussi vers l'Afrique du Nord. Cependant, au Proche Orient, "l'idée d'une légitimité califale en faveur de la famille proche du Prophète, incarnée dans Ali, fait des progrès" (A. Miquel, 1977). 
8 Uthmân, le troisième calife, est assassiné en 35/656 et après la bataille de Ciffîn en 37/657 Ali, vainqueur, propose la trêve à son adversaire Mu'awiya qui en profite pour étendre son pouvoir en Syrie et en Égypte. Les premiers schismatiques "ceux qui sortent" du cadre imposé à Ciffîn, les "Kharidjites", se révoltent et l'un d'eux assassine Ali en 40/661 auquel l'on reproche de n'avoir pas su s'imposer. Mu'awiya s'empare alors du califat et s'installe à Damas en laissant aux villes saintes leur rôle religieux (voir A. Miquel 1977).

9 C'est à partir de cette époque que les "puritains de l'islam", "violents et doctrinaires", contesteront sans relâche les pouvoirs établis. D'un côté les plus extrémistes, les Chîites, se représentent le chef de la communauté comme un guide inspiré par un décret divin en faveur de la descendance d'Ali, de l'autre la masse musulmane soucieuse d'unité communautaire et qui respectent la coutume, la tradition du Prophète, c'est-à-dire la Sunna, se définit comme sunnite.

Ce schisme chez les musulmans du Proche Orient allait devenir une brèche aux conséquences considérables. Tous les Berbères révoltés contre les nouveaux conquérants emboîtèrent le pas de tous les partis qui contestèrent l'autorité et l'organisation centralisée des califes. Le sens de leur révolte est double: ils veulent rester maîtres de leur territoire et s'affranchir de cette autorité étrangère sans toutefois rejeter la religion musulmane ; ils veulent garder la maitrise et les profits des échanges caravaniers avec le Soudan dont ils exploitent les trafics d'esclaves et de l'or en particulier. Les premières résistances héroïques menées par Kusayla*, puis la Kahina*, n'avaient pas l'étendue géographique et l'assise religieuse contestataire des mouvements kharidjites qui touchaient autant les populations du Proche Orient que celles du Maghreb.

11 Mais de plus, ces Berbères retrouvèrent dans les principes des doctrines religieuses et morales des Kharidjites leur propre conception de l'organisation de leur société où le chef est un guide garant de leurs règles plutôt qu'un souverain autoritaire (voir T. Lewicki 1983, p. 45 et O. Meunier 1997, p. 32). C'est là au demeurant la raison profonde qui a motivé les révoltes berbères au Maghreb contre toutes les autorités étrangères centralisatrices du pouvoir.

12 Deux sectes principales se sont partagé les dissidents au VIII siècle; ce sont: les Sufrites* qui représentaient la tendance la plus radicale et les Ibadites la tendance la plus modérée. Deux grandes révoltes marquèrent ce siècle au Maghreb : celle des Sufrites avec pour chefs Ukasa et Abdel Wahid al-Huwwarî, et celle menée par l'Ibadite Abû alKhattâb* (140/757). Puis au $\mathrm{x}^{\mathrm{e}}$ siècle la révolte organisée par Abû Yazîd*, Makhlad (331-5/943-7), le nukkarite surnommé "l'homme à l'âne" en raison de sa vie ascétique, ébranla le khalifat fatimide de l'Ifriqiya.

13 De ces trois grands mouvements, c'est certainement la révolte menée par Abû alKhattâb qui semble la mieux organisée et la plus importante. Car ce "porteur de la science" "hamalat al 'ilm", venu de Basra avec d'autres savants, organise avec l'aide des Huwwâra le premier État ibadite du Maghreb. Élu imam ibadite de Tripolitaine (757-758), Abû al-Khattâb s'empare de Tripoli, puis de Gabès et Kairouan en éliminant les Soufrites Warfajûma. La Tripolitaine, sous l'autorité des Ibadites, est alors le carrefour de toutes les voies terrestres d'ouest en est et du sud au nord (F. Belhachemi 1992, p. 28) et peut faire ainsi barrage aux Abassides. Mais après deux victoires contre les armées abassides, Abû-al-Khattâb vaincu est tué à Tawurgha en 761 . Son disciple “Abd al-Rahman Ibn Rustem (autre "porteur de la science") est désigné imam de Tahert 
(776-7). Bien qu'ayant perdu l'hégémonie sur le Maghreb de l'Est, les Huwwâra continuent leur lutte pour maintenir leur indépendance alors que l'imamat de Tahert se compromet avec les Aghlabides.

D'autres événements d'ordre stratégique interviennent alors à l'échelle de tout le continent. La grande piste allant de l'Égypte au Ghana qui traverse le Fezzan vers le Tchad, dont le relais principal est la ville de Zawîla, est interdite entre 881 et 884 sur ordre du souverain Ibn Tûlûn sous le prétexte des dangers qu'elle représente. En fait, cette décision du souverain tûlûnide vise l'activité commerciale des Ibadites Nafûsa en faisant basculer vers l'axe Sidjilmâsa/Ghâna la prépondérance des échanges nord-sud et vice versa. Les commerçants irakiens de Basra, de Kufa et des baghdadiens s'installent à Sidjilmâsa en délaissant Zawîla (voir J.-M. Cuoq 1985 qui traduit cette information d'Ibn Hawqal). Cette migration semble liée aux intérêts du royaume ibadite de Tahert hostile aux Nafûsa, car Basra (Bassorah), origine des Ibadites, a toujours soutenu ceux de Tahert dans leurs stratégies politico-économiques.

En conséquence, l'hégémonie des Nafûsa qui menaçaient les Aghlabides (délégués des Abassides) s'amoindrit en permettant à ces derniers de s'enrichir à nouveau dans leurs relations avec les 'Umayyades d'Espagne via Tahert et Sidjilmâsa. L'écrasement des Ibadites à l'est de Manu en 896 par les Aghlabides d'Ibrahim II est la conséquence de la trahison de Tahert à l'égard de ceux-ci. Cependant, à partir de 918 et avant la fin de la révolte d'Abu Yazid * en 947, les Huwwâra, qui forment "la dynastie des Banu Khattâb", redémarrent l'activité de Zawîla durant près de trois siècles (voir F. Belachemi 1992, p. 55 et 84). C'est l'époque de l'installation au Maghreb des Fatimides hostiles aux Huwwâra qui refluent vers le sud.

A cette époque, tous les échanges transsahariens sont encore sous le contrôle des Kharidjites. Mais ceux-ci n'ont plus la force militaire pour résister aux Fatimides (Chiîtes) au nord du Sahara. C'est alors que les Zirides (dynastie berbère) enrichis à l'ouest aux dépens des Fatimides rompent avec ceux-ci, se rallient au sunnisme (malékites) et reconnaissent le khalifat abasside de Baghdad. Pour les punir les Fatimides lancent les tribus nomades des Beni Hilal et Beni Soleim à l'assaut du Maghreb en 1050-52.

On assiste durant ce temps à la naissance et à la montée en puissance du mouvement almoravide (malékite) formé principalement de Berbères Sanhadja qui conquièrent d'abord le Ghana et convertissent sa population. Par la suite Tadmekka, conquise par le Ghana en 1083 avec l'aide des Almoravides, est ravie à l'autorité ibadite dans l'axe Ouargla/Tadmekka/Gao. Sur Tadmekka l'on possède le témoignage précieux d'Ibn Hawqal au milieu du $\mathrm{x}^{\mathrm{e}}$ siècle qui parle des Banu Tanmak, et d'Idrissi qui, au XII ${ }^{\mathrm{e}}$ siècle, signale les Azkâr à dix-huit étapes de Ghadamès, lesquels nomadisent au pied de la montagne "Tantanuh" (Al Idrissi 1968, p. 42).

Toutes les données géo-stratégiques du commerce caravanier saharien évoluent et changent alors pour ces multiples raisons. Les Huwwâra voient leur capitale Zawîla détruite par Karakuch (Caracoch-el-Ghozzi el-Modafferi ; voir Ibn Khaldoun, 1927, t. II, p. 91-92) vers 1190, au service du souverain d'Égypte Salah-ed-Dîn; le souverain du Kanem se convertit au malékisme. Le Sahara central et Ghadamès entrent définitivement dans l'échiquier des nouveaux itinéraires ; des migrations berbères vers l'Aïr et le Hoggar s'effectuent aussi durant ce XII ${ }^{\mathrm{e}}$ siècle (voir Djibo M. Hamani 1985). Mais tous les Berbères du groupe Huwwâra ne sont pas restés au Sahara. 

$\mathrm{XI}^{\mathrm{e}}$ siècle, et l'immensité des territoires qu'ils ont parcourus les ont affaiblis. Certains d'entre eux se sont mêlés aux autres Berbères tout en reconnaissant parfois la suprématie des diverses dynasties musulmanes de l'Afrique du Nord et de l'Espagne. Après les répressions des Fatimides au x ${ }^{\mathrm{e}}$ siècle et celle du hafside Abu Zakariyya au XIII ${ }^{\mathrm{e}}$ siècle, ils cessèrent de jouer un rôle politique en Ifriqîya. Ibn Kaldoun signale que les Huwwâra subsistent entre Barca et Alexandrie en Égypte, sur les plateaux de l'Ifriqîya depuis Tébessa jusqu'à Bédja et qu'ils se sont intégrés au sein des Béni Soleim “... auxquels du reste ils se sont assimilés par le langage et l'habillement ainsi que par l'habitude de vivre sous la tente. Comme eux, ils se servent de chevaux pour monture, ils élèvent des chameaux, ils se livrent à la guerre et ils font régulièrement la station du Tell dans l'été et celle du désert dans l'hiver. Ils ont oublié leur dialecte berbère pour apprendre la langue plus élégante des Arabes, et à peine comprennent-ils une seule parole de leur idiome" (Ibn Kaldoun, Histoire des Berbères, 1925, t. I, p. 278).

Mais il n'en va pas de même au sud, au Sahara central et au Sahel. Au pays de Gaugaua sont déjà installés les Lamta. Qui sont ces Lamta? Lamta se dit en berbère Ilemté ; ce vocable désigne aujourd'hui les habitants d'el Barkat près de Ghât et aussi au Touat et au Tidikelt des populations berbères que les Arabes appellent Touaregs (voir Foucauld, Dictionnaire touareg-français, III : 1087).

D'après une légende de l'Ahaggar, Lemtoûna serait la mère de tous les Touaregs ; celleci aurait eu une sœur, mère de la plupart des tribus berbères du Maroc et notamment des Berabers. L'on retrouve cette filiation chez Ibn Khaldoun sous une autre forme: Huwwâra, Sanhadja et Lamta seraient les descendants d'une même femme, "Tiski la boiteuse", mais issus de deux mariages successifs. Les Sanhadja, les Lamta, les Guezoula et les Heskoura seraient du même père, Acîl fils de Zéazâ, alors que les Huwwâra seraient descendants d'Aurigh-ibn-Bernès et issus d'un mariage précédent de Tiski (Ibn Khaldoun 1925,t. I, p. 273).

Les Lamta seraient donc des frères utérins des Huwwâra, qui auraient gardé le nom propre de leur tribu. Encore aujourd'hui, la plupart des Touaregs disent descendre de la même mère; la filiation du commandement chez beaucoup de groupes se faisait en ligne utérine et les Kel Ahaggar disent aujourd'hui avoir pour ancêtre celle qu'ils appellent Tin Hinane ("celle des tentes") dont le tombeau (daté du ve siècle) se situe près d'Abalessa; le squelette qui en a été exhumé était celui d'une femme qui devait boîter (voir sur Tin Hinan: M.-C. Chamla 1968; G. Camps in Zephyrus XXV, 1974, p. 497-517).

Ce qui nous intéresse ici c'est d'apprendre que ces Lamta qui faisaient partie d'un ensemble de tribus fuyant probablement devant une invasion arabe étaient déjà présents sur les rives du Niger au IX ${ }^{e}$ siècle et qu'ils semblent bien avoir précédé tous les autres Huwwâra. Seraient-ils les premiers Berbères ayant fui vers l'ouest, devant l'arrivée des Arabes et les conquêtes d'Uqba ibn Nafi'qui traversa le Fezzan et soumit le Kawar dès 666 ? où bien étaient-ils déjà familiers de ces régions avant les conquêtes arabes? Une pareille migration sur environ $2500 \mathrm{~km}$ ne peut se faire que lentement, avec une parfaite connaissance des itinéraires et des moyens de défense organisés. De plus, ces populations allaient vers une zone d'échanges stratégiques entre la boucle du Niger et le Maghreb où étaient déjà présents d'autres Berbères comme les Sanhadja d'origine orientale et les Saghmara d'origine zénète (voir M. Gast et S. Chaker 1979, p. 73-78). Leur installation dans cette zone ne faisait que renforcer la prédominance 
berbère sur les populations locales et où à cette époque les Arabes n'étaient pas encore présents. Cependant, cet ensemble berbère se réclamait toujours de l'islam; la marque la plus significative qu'ils en affichaient était le voilement dont ils avaient fait un attribut vestimentaire particulier exprimant leur communauté culturelle (ainsi que leur langue et leur organisation sociale). Car une des premières transformations physiques qu'ont dû imposer les conquérants arabes à ces populations sahariennes qui vivaient nu-tête, vêtues de peaux, mangeant exclusivement de la viande, des graines de cueillettes et vivant du lait de leurs troupeaux (voir Ibn Hawqal 1965, p. 99 et el Bekri 1913, p. 321 à propos des Almoravides), c'était de discipliner leur coiffure en portant un voile qui devait être à l'origine indigo (comme en portent encore aujourd'hui des populations yéménites). C'était en quelque sorte sortir de la sauvagerie pour entrer dans un monde civilisé, pourvu d'une mystique religieuse et d'une mission civilisatrice. Ces rudes guerriers montés à cheval ou sur des dromadaires qu'ils avaient très tôt, semble-t-il, dotés d'une selle originale extrêmement pratique placée sur le garrot de l'animal (que les Arabes n'ont jamais possédée), étaient redoutables et impressionnants derrière l'anonymat de leur voilement indigo, avec leurs cris de guerre suraigüs et des attaques surprises foudroyant leurs victimes.

Ces Berbères avaient compris que l'islam était désormais la référence incontournable des puissants et des conquérants mais ne voulaient pas pour autant se laisser arabiser, d'où leur choix d'un ibadisme berbère s'opposant au centralisme arabe. Ils défendaient par ailleurs les règles de transmission du pouvoir en matrilinéarité, coupant court aux tentatives de détournement du commandement de tout étranger se mariant à une femme berbère de haut rang (voir M. Gast 1976 et 1987). Ainsi défendus "du dedans", assurant la maîtrise d'immenses territoires, ces Berbères contrôlaient les flux d'échanges nord-sud/est-ouest dans la plus grande partie du Sahara jusqu'à l'arrivée des Européens à la fin du XIX ${ }^{e}$ siècle et au début du XXe siècle (voir Djibo M. Hamani 1989 sur l'expansion des Berbères au Sahara, chap. 2, p. 61).

Comment et dans quelles circonstances l'un des groupes Huwwâra s'est-il installé dans les massifs montagneux du Sahara central ? Nous devons remarquer que ces Dominants irréductibles, bien informés au niveau des stratégies politico-économiques et religieuses de l'Afrique et du Sahara, s'amoindrissaient en force et en nombre, et se trouvaient contraints d'investir des régions montagneuses parmi les plus pauvres, zones refuges où ils pouvaient assurer leur fuite et leur défense, mais aussi en se fractionnant en plusieurs groupes. Ainsi l'Ajjer, l'Ahaggar, l'Aïr, l'Adrar des Iforas devinrent leurs territoires de prédilection alors qu'ils guerroyaient souvent à plus de mille kilomètres de leur base pour garder leurs prérogatives sur les échanges caravaniers.

$\mathrm{Au}$ cours des $\mathrm{XIII}^{\mathrm{e}}$ et $\mathrm{XIV}^{\mathrm{e}}$ siècles, plusieurs groupes de Berbères issus des Huwwâra s'installent définitivement en Aïr (voir D.M. Hamani 1989, p. 73-88) mais aussi au Touat, au Gourara et au Tidikelt en dominant des populations d'agriculteurs qui leur servaient de relais. Le témoignage d'Ibn Battuta qui traversa le Hoggar en 1353 nous confirme la prédominance des Hukkâr (Kel Ahaggar) dans le contrôle des circuits caravaniers Sidjilmassa/Touat (Buda) - Takedda/Azelik. Car ceux-ci avaient ravi aux Imessufa (Messufa) l'autorité sur les mines de cuivre d'Azelik et le contrôle du commerce caravanier qui en découlait. Mais aucun témoignage ne signale l'Ahaggar avant le $\mathrm{xI}^{\mathrm{e}}$ siècle. C'est, semble-t-il, à partir du peuplement du Tidikelt au début du XIV siècle que les Huwwâra prennent position au Sahara central. 

politiques et religieuses nord-sud et est-ouest durant dix siècles. Si, par l'exploitation systématique des textes des historiens arabes ayant relaté les événements qu'ils rapportent du $\mathrm{VII}^{\mathrm{e}}$ au XVIII ${ }^{\mathrm{e}}$ siècle, l'on peut désormais reconstituer les grands moments de cette histoire, les Berbères eux-mêmes n'ont pas laissé de textes expliquant leur point de vue et leurs choix stratégiques. Cette absence de mémoire écrite a été de tout temps leur grande faiblesse face à des États organisés qui, quels que soient les avatars des différents pouvoirs s'étant succédé, ont maintenu le souci de leurs intérêts propres, à travers la mémoire de leur Histoire. Celle des Berbères est constituée de bribes de souvenirs, de légendes, d'anecdotes, de contes et de poèmes qui représentent leur patrimoine le plus précieux transmis par leur littérature orale. Le travail de collecte de tout cet héritage ne fait que commencer. Il sera très important pour la survie de la culture amazigh en général et touarègue en particulier d'y prêter soutien et attention.

\section{BIBLIOGRAPHIE}

AL IDRISI, Description de l'Afrique et de l'Espagne, trad. R. Dozy et M.-J. de Goeje, Leyde, 1866 ; réimpr. 1968, 393 p. + texte arabe.

AL YAKUBI, Kitab al Buldan, trad. Gaston Wiet, “les pays”, Le Caire, 1937. 
BARTH H., Voyages et découvertes dans l'Afrique septentrionale et centrale, 4 tomes, Paris, Firmin Didot, 1863.

BELHACHEMI F., Nouvelle interprétation du processus de peuplement dans le massif du Hoggar à partir des géographes arabes, Revue de Géographie alpine, t. LXXIX, 1, 1991 (numéro spécial Montagnes du Sahara), p. 143-164.

BELHACHEMI F., Anthropologie économique et historique des Touareg du Hoggar. Thèse pour le doctorat d'anthropologie sous la dir. de P. Ph. Rey, novembre 1992, 452 p. + annexes (453 à 573).

BENHAZERA M., Six mois chez les Touareg du Ahaggar, Alger, typographie Adolphe Jourdan, 1908, 233 p. BOURGEOT A., La formation des classes sociales chez les Twareg de l'Ahaggar, Cahiers du CERM, $\mathrm{n}^{\circ}$ 121, 1975, Études sur les sociétés de pasteurs nomades, classes sociales et état dans les sociétés. CAMPS G., Le tombeau de Tin Hinan à Abalessa, Travaux de l'Institut de Recherches sahariennes, $\mathrm{t}$. XXIV, $1^{\text {er }}$ et $2^{\text {e }}$ sem. 1965 , p. $65-83$.

CAMPS G, L'âge du tombeau de Tin Hinan, ancêtre des Touaregs du Hoggar, Zephyrus 25, 1974, p. 497-516.

CHAMLA M.-C, Les populations anciennes du Sahara et des régions limitrophes, Mém. du CRAPE IX, Paris, AMG, 1968, $248 \mathrm{p}$.

Cuoq (J.-M.), Histoire de l'islamisation de l'Afrique de l'Ouest, des origines à la fin du XVI siècle, Paris, Geuthner, 1984, 347 p.

CUOQ J.-M., Recueil des sources arabes concernant l'Afrique occidentale du VIII au XVI siècle, préf. de R. Mauny, Paris, CNRS, 1985, 490 p.

DEVISSE J., Routes de commerce et échanges en Afrique occidentale en relation avec la Méditerranée (Un essai sur le commerce africain médiéval du XI ${ }^{\mathrm{e}}$ au XVI ${ }^{\mathrm{e}}$ siècle), Revue d'Histoire économique et sociale, 1972, vol. 1 (p. 42-73), vol. 2 (p. 357-397).

DUVEYRIER H., Les Touareg du Nord, Paris, Challamel Aîné, 1864, 499 p. El Bekri, Description de l'Afrique septentrionale, trad. M. G. de Slane, Alger, 1913, A. Jourdan, 405 p.

FOUCAULD, Père Charles de, Dictionnaire touareg-français, Paris, Impr. Nat. 1951-1952, 4 vol.

GAST M., Les Kel Rela : historique et essai d'analyse du groupe de commandement des Kel Ahaggar, Revue de l'Occident musulman et de la Méditerranée, 1976, n² 21, p. 47-63.

GAST M., Pastoralisme nomade et pouvoir : la société traditionnelle des Kel Ahaggar, in Production pastorale et société, Paris, Maison des Sciences de l'Homme/Cambridge, 1979, p. 201-220.

GAST M. et CHAKER S., Sagmara, Saghmara et Isseqqamarènes à travers l'histoire du Sahara central, in Recherches sahariennes, 1. Programme Marges désertiques, Paris, CNRS, 1979, p. 79-81.

GAST M., Les verrous secrets d'une société indépendante au Sahara central : règles d'héritage et transmission des biens chez les Kel Ahaggar, in Hériter en pays musulman, Habous, Lait vivant, Manyahuli, sous la dir. de M. Gast, Paris, Ed. du CNRS, 1987, p. 169-189.

HAMANI DJBO M., Au carrefour du Soudan et de la Berbérie : le sultanat touareg de l'Ayar, Études nigériennes $\mathrm{n}^{\circ}$ 55, Niamey, I.R.S.H., 1989, 522 p.

IBN BATTUTA, Voyages d'Ibn Batouta, trad. Defremery C. et Sanguinetti B.R. (1853-58), Paris, Maspero, 1982. 
IBN HAUQAL, Configuration de la terre (Kitâb surat al - Ard), introd. et trad, par J.-H. Kramers et G. Wiet, 2 tomes, Beyrouth, Com. intern, pour la trad, des chefs-d'œuvre ; Paris, Maisonneuve et Larose, 1964.

IBN KHALDOUN, Histoire des Berbères et des dynasties musulmanes de l'Afrique septentrionale, trad, de Slane, 4 vol., Paris, Geuthner, 1925-1956.

IDRIS H.R., La Berbérie orientale sous les Zirides, $X^{e}$-XII siècles, Paris, Adrien Maisonneuve, 1962.

IDRISSI (al-Idrîsî 1100-1162), Description de l'Afrique et de l'Espagne, trad, de Dozy et de Goeje, Leyde, E.-J. Brill, 1866.

Jean-Léon L'AFRICAIN, Description de l'Afrique, nouvelle édition traduite de l'italien par A. Epaulard et annotée par A. Epaulard, Th. Monod, H. Lhote et R. Mauny, Paris, Lib.d'Amérique et d'Orient Adrien Maisonneuve, 1956, 2 vol.

JULIEN Ch.-A., Histoire de l'Afrique du Nord, Paris, Payot, 1931, 866 p.

LEWICKI T., L'État nord-africain de Tâhert et ses relations avec le Soudan occidental à la fin du VIII siècle et au IX ${ }^{\mathrm{e}}$ siècle, Cahier d'Études africaines, $\mathrm{n}^{\circ}$ 8, 1962, vol. II, $4^{e}$ cahier, p. 513-535.

LEWICKI T., Études maghrébines et soudanaises II, Varsovie, 1983, Editions scientifiques de Pologne.

LEWICKI T., Hawwâra, Encyclopédie de l'Islam III, p. 305-309.

MARTIN A.G.P., Les oasis sahariennes (Gourara, Touat, Tidikelt), Paris, Augustin Challamel édit., 1908, $406 \mathrm{p}$.

MARTY P., Études sur l'islam et les tribus du Soudan (coll. de la Revue du monde musulman), Paris, Leroux éd., 1920, 4 vol.

MEUNIER O., Les routes de l'islam. Anthropologie politique de l'islamisation de l'Afrique de l'Ouest en général et en pays hawsa en particulier du VIII au XIX siècle, Paris, L'Harmattan, 1997, 203 p.

MIQUEL A., L'Islam et sa civilisation, VII-XX ${ }^{e}$ siècle, Paris, A. Colin, 1977, 600 p.

NORRIS H.T., The Tuaregs. Their islamic legacy and its diffusion in the Sahel, Wilts, England, 1975.

INDEX

Mots-clés : Population/peuplement 